FTG-114-USU

February 2008

\title{
Symmetries of the Einstein Equations
}

\author{
C. G. Torre \\ Department of Physics \\ Utah State University \\ Logan, UT 84322-4415 \\ USA \\ I. M. ANDERSON \\ Department of Mathematics and Statistics \\ Utah State University \\ Logan, UT 84322-3900 \\ USA
}

\begin{abstract}
Generalized symmetries of the Einstein equations are infinitesimal transformations of the spacetime metric that formally map solutions of the Einstein equations to other solutions. The infinitesimal generators of these symmetries are assumed to be local, i.e., at a given spacetime point they are functions of the metric and an arbitrary but finite number of derivatives of the metric at the point. We classify all generalized symmetries of the vacuum Einstein equations in four spacetime dimensions and find that the only generalized symmetry transformations consist of:
\end{abstract}

(i) constant scalings of the metric

(ii) the infinitesimal action of generalized spacetime diffeomorphisms.

Our results rule out a large class of possible "observables" for the gravitational field, and suggest that the vacuum Einstein equations are not integrable. 


\section{Introduction}

A point symmetry of a system of differential equations is a one-parameter group of transformations of the underlying space of independent and dependent variables that carries solutions of the equations to other solutions. Over a century ago, Lie [1] initiated a geometrical approach to the study of differential equations based on their point symmetries. By considering infinitesimal group transformations, Lie produced algorithms for finding the point symmetries of any system of equations. For differential equations derived from a variational principle, Noether [2] proved that those point symmetries which preserve the action lead to conservation laws. Noether also pointed out that not all conservation laws arise as a consequence of point symmetries. She therefore introduced the idea of derivative-dependent infinitesimal symmetry transformations, now known as "generalized symmetries". Her work, together with the appropriate technical assumptions [3], establishes a one-to-one correspondence between generalized symmetries of the underlying action functional and conservation laws.

In recent years, symmetry analysis has become an important tool in the study of differential equations $[3,4,5]$. This is due, in part, to the intimate connection between generalized symmetries and integrable systems of partial differential equations. Indeed, a widely acknowledged attribute of an integrable field theory is the existence of an infinite set of generalized symmetries [6,7]. Physically, the importance of symmetries of field equations stems from their use in: classifying solutions to the equations, construction of solution generating algorithms, and, via Noether's theorem, the identification of conservation laws.

While applied mathematicians have devoted a large amount of attention to applications of the theory of symmetry groups to a variety of non-linear partial differential equations, relatively few results have been obtained for the most important non-linear field equations of theoretical physics, e.g., the Yang-Mills equations and the Einstein equations. The purpose of this letter is to report on the results of a generalized symmetry analysis of 
the vacuum Einstein equations in four spacetime dimensions. Our analysis has yielded a complete classification of the generalized symmetries.

Why look for generalized symmetries of the Einstein equations? The existence of "hidden symmetries" of the Einstein equations would lead to solution generating/classification techniques, and perhaps even information about the "general solution" to the Einstein equations. There are hints that such symmetries may exist: the two Killing field reduction of the Einstein equations leads to an integrable system of partial differential equations [8,9]; the self-dual Einstein equations exhibit an infinite number of symmetries and can be integrated using twistor methods $[10,11]$. Recently, a set of generalized symmetries has been presented for the Einstein equations using the Newman-Penrose formalism [12]. A complete generalized symmetry analysis provides a systematic and rigorous way to unravel some aspects of the integrable behavior of the gravitational field equations. In particular, such an analysis indicates whether the rich structure of special reductions of the Einstein equations extends to the full theory, as well as clarifying the status of the symmetries of [12]. An equally important consequence of a generalized symmetry analysis stems from the fact that the existence of generalized symmetries of the Einstein equations is a necessary condition for the existence of local differential conservation laws for the gravitational field. If such symmetries/conservation laws could be found, they would lead to "observables" for the gravitational field. It has long been an open problem in relativity theory to exhibit such observables. The lack of observables currently hampers progress in canonical quantization of general relativity [13].

\section{Generalized Symmetries of the Einstein Equations}

A generalized symmetry of the Einstein equations $G_{a b}=0$ is an infinitesimal transformation $\delta g_{a b}$ of the metric which formally maps solutions of the Einstein equations to other "near by" solutions. The generator of a generalized symmetry transformation is built from the spacetime position $x$, the metric, and an arbitrary but finite number of derivatives of 
the metric at $x$ :

$$
\delta g_{a b}=h_{a b}\left(x, g, \partial_{x} g, \ldots\right) .
$$

We say that the functions $h_{a b}$ generate a symmetry if and only if they satisfy the linearized Einstein equations,

$$
\left(-g^{d c} \delta_{m}^{(a} \delta_{n}^{b)}-g^{a b} \delta_{m}^{(d} \delta_{n}^{c)}+2 g^{d b} \delta_{m}^{(c} \delta_{n}^{a)}\right) \nabla_{d} \nabla_{c} h_{a b}=0
$$

when the Einstein equations $G_{a b}=0$ hold. In (5) $\nabla_{a}$ is the unique connection compatible with the Einstein metric $g_{a b}$.

Note that while the infinitesimal transformation is a local function of the metric, the finite transformation it generates (if it exists) can be quite non-local. This is because the one-parameter family of solutions $g_{a b}(\lambda)$ generated by $h_{a b}$ is obtained by solving a system of partial differential equations

$$
\frac{\partial g_{a b}}{\partial \lambda}=h_{a b}\left(x, g, \partial_{x} g, \ldots\right) .
$$

There are two classes of generalized symmetries that can be identified immediately. The first is the well-known scale symmetry of the Einstein equations, which corresponds to the infinitesimal point symmetry

$$
\delta g_{a b}=c g_{a b}
$$

where $c$ is a constant. The second symmetry stems from the general covariance (diffeomorphism covariance) of the Einstein equations. It is well-known that, for each vector field $V^{a}(x)$, the tensor

$$
\delta g_{a b}=\nabla_{a} V_{b}+\nabla_{b} V_{a}
$$

satisfies the linearized equations (5) when $G_{a b}=0$. This point symmetry reflects the general covariance of the Einstein equations. The symmetries (1a), (1b) comprise all the 
point symmetries of the vacuum Einstein equations [14]. Given a generalized (covariant) vector field $X_{a}=X_{a}\left(x, g, \partial_{x} g, \ldots\right)$, a direct computation shows that

$$
\delta g_{a b}=\nabla_{a} X_{b}+\nabla_{b} X_{a}
$$

also satisfies the linearized Einstein equations when $G_{a b}=0$, and is hence a generalized symmetry. Let us call the symmetry (2) a generalized diffeomorphism symmetry. According to the principles of general relativity, one should really view the Einstein equations as a set of partial differential equations that determine diffeomorphism equivalence classes of metrics. Thus the generalized diffeomorphism symmetry (2) is considered physically trivial.

Our symmetry analysis shows that the scale symmetry (1a) and generalized diffeomorphism symmetry (2) are in fact the only generalized symmetries admitted by the vacuum Einstein equations. The proof of this fact is rather long, accordingly it is best to begin by classifying an important sub-class of symmetries, the "natural" generalized symmetries. Natural symmetries are generated by those $h_{a b}$ which transform properly under spacetime diffeomorphisms. Specifically, a "natural tensor" $h_{a b}$ is a tensor which is built from the metric, the curvature, and covariant derivatives of the curvature up to some finite order $[15,16,17]$. Such $h_{a b}$ are universal geometric expressions, and are defined on any manifold irrespective of its topological structure. In this case we have the following theorem.

Theorem 1: Let $\delta g_{a b}=h_{a b}\left(g, \partial_{x} g, \ldots\right)$ be a natural generalized symmetry for the Einstein equations $G_{a b}=0$ in four spacetime dimensions. Then

$$
h_{a b}=c g_{a b}+2 \nabla_{(a} X_{b)},
$$

where $c$ is a constant and

$$
X_{a}=X_{a}\left(g, \partial_{x} g, \ldots\right)
$$

is a natural (covariant) vector field. 
We will now sketch the proof of this theorem; a more rigorous discussion with all of the details will be given elsewhere [18]. All our considerations are local in spacetime, i.e., we do not worry about boundary conditions, so our results are quite general. The strategy is to view $h_{a b}$ as a collection of functions on a very large space $\mathcal{J}^{N}$ parametrized by the spacetime point $x$, the metric, the curvature, and the first $N$ covariant derivatives of the curvature at $x$. Note that $\mathcal{J}^{N}$ is finite dimensional. For example, $\mathcal{J}^{0}$ is the 34 -dimensional space parametrized by $\left(x, g_{a b}, R_{a b c d}\right)$, where $R_{a b c d}$ is the curvature tensor. The Einstein equations $G_{a b}=0$, and their derivatives $\nabla_{c} G_{a b}=0, \nabla_{c} \nabla_{d} G_{a b}=0, \ldots$, define a subspace $\mathcal{E}^{N}$ of $\mathcal{J}^{N}$ and it is only on this subspace that the equations (5) need to be satisfied. In the example above, $\mathcal{E}^{0}$ is a 24 -dimensional submanifold of $\mathcal{J}^{0}$ defined by the equations

$$
g^{a c} R_{a b c d}=0
$$

Using the chain rule, the linearized Einstein equations (5) become an overdetermined system of partial differential equations restricting the functional form of $h_{a b}$. Because the resulting system of equations is quite overdetermined, it is possible to find all of its solutions.

The primary complication in the analysis is that (5) need only be satisfied on $\mathcal{E}^{N}$. To handle this complication we appeal to work of Penrose $[19,20]$ which gives coordinates for $\mathcal{E}^{N}$ in terms of spinors. The introduction of two-component spinors at this point restricts our analysis to the conventional four-dimensional spacetime of Einstein's general theory of relativity. While it is quite possible that our results generalize to higher dimensions, as it stands our analysis is limited to the four-dimensional case.

Penrose's "exact set of fields" for the vacuum Einstein equations $[19,20]$ can be viewed as giving an explicit parametrization of $\mathcal{E}^{N}$. Let $\Psi_{A B C D}$ represent the Weyl spinor (capital Latin indices are two-component spinor indices). Penrose's result is that the symmetrized 
covariant derivatives of the Weyl spinor

$$
\Psi_{A_{1} \cdots A_{n+4}}^{A_{1}^{\prime} \cdots A_{n}^{\prime}}:=\nabla_{\left(A_{1}\right.}^{\left(A_{1}^{\prime}\right.} \cdots \nabla_{A_{n}}^{\left.A_{n}^{\prime}\right)} \Psi_{\left.A_{n+1} A_{n+2} A_{n+3} A_{n+4}\right)}
$$

and its complex conjugate, for $n=0,1, \ldots$, are freely specifiable at a point of an Einstein space and completely determine the curvature and all covariant derivatives of the curvature at that point. We will denote the spinor (11) and its complex conjugate by $\Psi^{n}$ and $\bar{\Psi}^{n}$ respectively. Using the spinor form of the Ricci and Bianchi identities on an Einstein space it is straightforward to show that $\Psi^{n}$ satisfies

$$
\nabla_{B}^{B^{\prime}} \Psi_{A_{1} \cdots A_{n+4}}^{A_{1}^{\prime} \cdots A_{n}^{\prime}}=\Psi_{A_{1} \cdots A_{n+4} B}^{A_{1}^{\prime} \cdots A_{n}^{\prime} B^{\prime}}+\mathcal{Q}
$$

where $\mathcal{Q}$ denotes terms involving $\Psi^{k}$ and $\bar{\Psi}^{k}$ for $k=0, \ldots, n-1$.

The spinor translation of the generalized symmetry equation (5) can be put into the form:

$$
\begin{gathered}
{\left[-\epsilon^{A B} \epsilon^{A^{\prime} B^{\prime}} \epsilon_{M R} \epsilon_{N S} \delta_{R^{\prime}}^{M^{\prime}} \delta_{S^{\prime}}^{N^{\prime}}\right.} \\
\left.+\delta_{M}^{A} \epsilon^{A^{\prime} N^{\prime}}\left(\epsilon_{N S} \delta_{R}^{B} \delta_{R^{\prime}}^{B^{\prime}} \delta_{S^{\prime}}^{M^{\prime}}+\epsilon_{N R} \delta_{S}^{B} \delta_{S^{\prime}}^{B^{\prime}} \delta_{R^{\prime}}^{M^{\prime}}\right)\right] \\
\times \nabla_{A A^{\prime}} \nabla_{B B^{\prime}} h_{M^{\prime} N^{\prime}}^{M N}=0
\end{gathered}
$$

where $h_{M^{\prime} N^{\prime}}^{M N}$ is the spinor form of the symmetry generator $h_{a b}$. We have also introduced the skew-symmetric $\epsilon$-spinors, which are used to raise and lower spinor indices. Equation (10) is to be satisfied modulo the Einstein equations. Hence, without loss of generality, we can assume in (10) that $h_{M^{\prime} N^{\prime}}^{M N}$ is a function of the soldering form $\sigma_{a}^{A A^{\prime}}$, where

$$
g_{a b}=\sigma_{a}^{A A^{\prime}} \sigma_{b A A^{\prime}}
$$

and the $\Psi^{n}, \bar{\Psi}^{n}$-spinors up to some finite derivative order $N$. The natural generalized symmetry $h_{M^{\prime} N^{\prime}}^{M N}$ must satisfy (10) for all values of the $\Psi^{n}$ spinors and their complex conjugates.

As an illustration of our analysis let us assume that $N=1$ so that $h_{a b}$ is a natural tensor depending on no more than three derivatives of the metric at a given point:

$$
h_{a b}=h_{a b}\left(\sigma, \Psi^{0}, \bar{\Psi}^{0}, \Psi^{1}, \bar{\Psi}^{1}\right) .
$$


Because (10) involves two derivatives of $h_{a b}$, the left-hand side of (10) is a function of the soldering form and $\Psi^{n}, \bar{\Psi}^{n}$ - spinors for $n=0, \ldots, 3$. Differentiating (10) with respect to $\Psi^{3}$ leads to the following restriction on the dependence of $h_{a b}$ on the $\Psi^{1}$ - spinors:

$$
h_{M^{\prime} N^{\prime} E^{\prime}}^{M(N A B C D E)}+h_{E^{\prime} N^{\prime} M^{\prime}}^{M(N A B C D E)}=0,
$$

where we have defined

$$
h_{M^{\prime} N^{\prime} E^{\prime}}^{M N A B C D E}:=\frac{\partial h_{M^{\prime} N^{\prime}}^{M N}}{\partial \Psi_{A B C D E}^{E^{\prime}}} .
$$

Differentiating (10) with respect to $\bar{\Psi}^{3}$ shows that the complex conjugate of (12) holds also.

Next, let us demand that the second derivative of (10) with respect to $\Psi^{2}$ vanish for all values of the $\Psi^{n}$-spinors. Using (12) this leads, after some analysis, to

$$
\frac{\partial^{2} h_{M^{\prime} N^{\prime}}^{M N}}{\partial \Psi_{A B C D E}^{E^{\prime}} \partial \Psi_{R S T U V}^{V^{\prime}}}=0 .
$$

Thus $h_{a b}$ is linear in its dependence on $\Psi^{1}$. Similar computations involving second derivatives of (10) with respect to $\Psi^{2}, \bar{\Psi}^{2}$ and $\bar{\Psi}^{2}, \bar{\Psi}^{2}$ show that the symmetry must be linear in its dependence on the $\bar{\Psi}^{1}$-spinors, and also that $h_{a b}$ contains no terms involving products $\bar{\Psi}^{1} \Psi^{1}$.

We have thus found that the spinor expression of the generalized symmetry $h_{a b}$ takes the form

$$
h_{M^{\prime} N^{\prime}}^{M N}=A_{M^{\prime} N^{\prime} E^{\prime}}^{M N A B C D E} \Psi_{A B C D E}^{E^{\prime}}+B_{M^{\prime} N^{\prime}}^{M N}+\text { complex conjugate, }
$$

where the $A$ and $B$ spinors depend on the soldering form and the undifferentiated Weyl spinors $\Psi^{0}, \bar{\Psi}^{0}$. The condition (12) gives us further information about the spinor $A ;(12)$ is satisfied if and only if there exist spinors $D$ and $X$ such that

$$
A_{M^{\prime} N^{\prime} E^{\prime}}^{M N A B C}=\epsilon^{M(A} D_{M^{\prime} N^{\prime} E^{\prime}}^{C D E} \epsilon^{B) N}+X_{M^{\prime}}^{M(B C D E} \epsilon^{A) N} \delta_{E^{\prime}}^{N^{\prime}}+X_{N^{\prime}}^{N(B C D E} \epsilon^{A) M} \delta_{E^{\prime}}^{M^{\prime}} .
$$


We further restrict the structure of the $A$-spinor by taking the mixed second partial derivative of (10) with respect to $\Psi^{2}$ and $\Psi^{1}$. After considerable analysis, the resulting equations can be shown to imply: (i) the $D$-spinor in (13) is independent of $\Psi^{0}$ and $\bar{\Psi}^{0}$, i.e., $D$ is a function of the soldering form only; and (ii) the spinor $X$ is a gradient with respect to $\Psi^{0}$, i.e.,

$$
X_{M^{\prime}}^{M B C D E}=\frac{\partial X_{M^{\prime}}^{M}}{\partial \Psi_{B C D E}}
$$

Here $X_{M}^{M^{\prime}}$ is the spinor form of a natural spacetime vector field.

Given (14), the $X$-spinors correspond to a generalized diffeomorphism symmetry (2). This can be seen by comparing the coefficient of the $\Psi^{1}$ term in the spinor form of (2), namely

$$
\nabla_{A}^{A^{\prime}} X_{B}^{B^{\prime}}+\nabla_{B}^{B^{\prime}} X_{A}^{A^{\prime}}=\frac{\partial X_{B}^{B^{\prime}}}{\partial \Psi_{R S T U}} \Psi_{R S T U A}^{A^{\prime}}+\frac{\partial X_{A}^{A^{\prime}}}{\partial \Psi_{R S T U}} \Psi_{R S T U B}^{B^{\prime}}+\text { complexconjugate }
$$

with the last two terms of (13).

To summarize, we have found that the only generalized symmetry for $N=1$ is a linear function of $\Psi^{1}$. Modulo terms of the form (2), the coefficient $A$ of the $\Psi^{1}$ term is a natural spinor built from the soldering form only. However, it can be shown that there is no spinor with the symmetries of $A$ that is built solely from the soldering form. Therefore, modulo generalized diffeomorphism symmetries, the linear term in $\Psi^{1}$ vanishes and we conclude that (modulo (2)) the symmetry can only depend on $\Psi^{0}, \bar{\Psi}^{0}$ and the soldering form:

$$
h_{a b}=2 \nabla_{(a} X_{b)}+h_{a b}^{\prime}\left(\sigma, \Psi^{0}, \bar{\Psi}^{0}\right)
$$

We now repeat the whole analysis starting with (10) and ending with (14) under the assumption that the symmetry only depends on the soldering form and the undifferentiated Weyl spinors. A virtually identical series of calculations proves that $h_{a b}^{\prime}$ is a function of the soldering form only, $h_{a b}^{\prime}=h_{a b}^{\prime}(\sigma)$. This is easily seen to imply that the point symmetry 
$h_{a b}^{\prime}$ can only be the scaling symmetry (1a), i.e., $h_{a b}^{\prime}=c g_{a b}$. This proves the theorem when $N=1$.

The proof of Theorem 1 in general is again via induction in the dependence of the symmetry $h_{a b}$ on derivatives of the metric. The spinor equations that arise in the analysis for $N>1$ are considerably more complicated than in the example above, but they can be solved using elementary spinor techniques.

Theorem 1 still leaves open the possibility that a non-trivial generalized symmetry can be constructed in some non-universal way from the metric, say with the aid of some externally prescribed auxiliary fields. Such symmetries are not universal in the sense that their existence may depend on the underlying manifold structure of the spacetime. While it is hard to know a priori what the geometrical meaning of such a symmetry could be, if we view the Einstein equations as just some set of partial differential equations, and ignore their geometric structure, then it is natural to seek such symmetries. We do this by allowing the symmetry generator $h_{a b}$ to be an explicit function of the spacetime position $x$ and drop the assumption that it is built only from the metric, curvature and covariant derivatives of curvature. Thus $h_{a b}$ is now allowed to depend on the spacetime position, the metric and a finite number of derivatives of the metric at a point, with no tensoriality assumptions. It is possible to generalize the analysis that leads to Theorem 1 to this case, and we can prove the more general theorem [18]:

Theorem 2 : Let $\delta g_{a b}=h_{a b}\left(x, g, \partial_{x} g, \ldots\right)$ be a generalized symmetry for the Einstein equations $G_{a b}=0$ in four spacetime dimensions. Then

$$
h_{a b}=c g_{a b}+2 \nabla_{(a} X_{b)}
$$

where $c$ is a constant and

$$
X_{a}=X_{a}\left(x, g, \partial_{x} g, \ldots\right)
$$


The proof of Theorem 2 involves the enlargement of the spinor variables to include the non-tensorial parts of the metric derivatives. An inductive proof, similar to that used in the natural case, is used to reduce the derivative dependence of the symmetry generator to only first derivatives of the metric modulo terms of the form (2). Because of the complicated dependence of the linearized Einstein equations on first and second derivatives of the metric occurring in the covariant derivatives, this case - first-order generalized symmetries - must be treated separately. A lengthy analysis leads to the result that, modulo the generalized diffeomorphism symmetry, the symmetry generator is a function of the undifferentiated metric only. This leads back to the scale symmetry and completes the proof.

Theorems 1 and 2 allow us to determine the structure of possible conservation laws for the vacuum Einstein equations. Let us define a local differential conservation law for the Einstein equations as a current (vector density) $J^{a}=J^{a}\left(x, g, \partial_{x} g, \ldots\right)$ satisfying

$$
\nabla_{a} J^{a}=\partial_{a} J^{a}=0,
$$

when $G_{a b}=0$. Then we can prove the following corollary.

Corollary 1: Let $J^{a}$ be a local differential conservation law for the vacuum Einstein equations in four spacetime dimensions. Then there exists functions $S^{a b}\left(x, g, \partial_{x} g, \cdots\right)$ skew symmetric on $a$ and $b, S^{a b}=-S^{b a}$, such that, up to terms that vanish when the Einstein equations hold,

$$
J^{a}=\nabla_{b} S^{a b} .
$$

If $J^{a}$ is a natural vector density, i.e., built from the metric, curvature and covariant derivatives of the curvature, then $S^{a b}$ can be chosen to be a natural tensor density.

This corollary follows from the fact that the existence of a generalized symmetry is a necessary condition for the existence of a local differential conservation law (see, e.g., [3]), and some fundamental results from the theory of the variational bicomplex [21]. From the point of view of the theory of local differential conservation laws, the form (9) of $J^{a}$ 
is trivial in the sense that such conservation laws are always possible for any system of equations, irrespective of the form of the equations. Nevertheless, such currents can have a physical role to play in general relativity. Indeed, (9) forms the basis for constructing energy-momentum pseudo-tensors for the gravitational field [22].

The form (9) of the conservation laws has strong implications for the existence of "observables" in the Hamiltonian formulation of gravitation in closed universes. Recall that observables are defined as functions on the gravitational phase space that have weakly vanishing Poisson brackets with the super-Hamiltonian and super-momentum [23,24]. This is equivalent to defining observables as constants of motion for the Einstein equations. From (9), however, it is clear that if the spatial manifold is compact without boundary there can be no non-trivial constants of motion built as spatial integrals of local functions of the spacetime metric and its derivatives. Thus our generalized symmetry analysis has ruled out a large class of observables. In particular, we conjecture that there are no observables built as spatial integrals of local functions of the canonical (ADM) coordinates and momenta (and their derivatives). It would thus appear that observables must be constructed in a non-local fashion, e.g., along the lines of those found in the class of cylindrically symmetric Einstein metrics in [25]. We are currently exploring the Hamiltonian implications of our analysis.

\section{Summary}

We have classified all generalized symmetries and local differential conservation laws of the vacuum Einstein equations in four spacetime dimensions. The symmetries consist of constant scalings and the induced action of infinitesimal generalized diffeomorphisms. The corresponding conservation laws are trivial. We note that the vacuum Einstein equations, when viewed as a system of equations for diffeomorphism equivalence classes of metrics, fail to pass a widely acknowledged "litmus test" for the integrability of a system of partial differential equations, namely, the existence of an infinite-dimensional set of generalized 
symmetries $[6,7]$.

Our analysis suggests several questions for further study; they include: the Hamiltonian

interpretation of Theorems 1,2 and Corollary 1, existence of generalized symmetries of subsystems of the Einstein equations, existence of non-local symmetries, e.g., Backlund transformations, and existence of generalized symmetries of the Einstein equations with matter couplings.

\section{Acknowledgments}

This material is based upon work supported by the National Science Foundation under Grant No. DMS-9100674 (IMA), and a Utah State University Faculty Research Grant (CGT).

\section{REFERENCES}

1. S. Lie, Geometrie der Beruhrungstransformationen, (B. G. Teubner, Leipzig 1896).

2. E. Noether, Nachr. Konig. Gesell. Wissen. Gottinger Math. Phys. Kl. , 235 (1918).

3. P. Olver, Applications of Lie Groups to Differential Equations, (Springer-Verlag, New York 1986).

4. G. Bluman and S. Kumei, Symmetries of Differential Equations, (Springer-Verlag, New York 1989).

5. L. Ovsiannikov, Group Analysis of Differential Equations, (Academic Press, New York 1982).

6. A. Fokas, Stud. Appl. Math. 77, 253 (1987). 
7. A. Mikhailov, A. Shabat, and V. Sokolov in What is Integrability?, ed. V. Zakharov (Springer-Verlag, New York 1991).

8. V. Belinsky and V. Zakharov, Sov. Phys. JETP 50, 1 (1979).

9. I. Hauser and F. Ernst, J. Math. Phys. 22, 1051 (1981).

10. R. Penrose, Gen. Rel. Grav. 7, 31 (1976).

11. C. Boyer and P. Winternitz, J. Math. Phys. 30, 1081 (1989).

12. M. Gurses, Phys. Rev. Lett. 70, 367 (1993).

13. C. Rovelli and L. Smolin, Nucl. Phys. B331, 80 (1990).

14. N. Ibragimov, Transformation Groups Applied to Mathematical Physics, (D. Reidel, Boston 1985).

15. T. Y. Thomas, Differential Invariants of Generalized Spaces, (Cambridge University Press, Cambridge 1934).

16. D. Epstein, J. Diff. Geom. 10, 631 (1975).

17. I. M. Anderson, Ann. Math. 120, 329 (1984).

18. I. M. Anderson and C.G. Torre, in preparation 1993.

19. R. Penrose, Ann. Phys. 10, 171 (1960).

20. R. Penrose and W. Rindler, Spinors and Space-Time, Vol. 1, (Cambridge University Press, Cambridge 1984).

21. I. M. Anderson, "Introduction to the Variational Bicomplex", in Mathematical Aspects of Classical Field Theory (Eds. M. Gotay, J. Marsden, V. Moncrief), Cont. Math. 32, 51 (1992). 
22. J. Goldberg in General Relativity and Gravitation: 100 Years After the Birth of Albert Einstein, Vol. 1, edited by A. Held (Plenum, New York 1980).

23. K. Sundermeyer, Constrained Dynamics, (Springer-Verlag, Berlin 1982).

24. Kuchař has shown there are no non-trivial observables that are linear functionals of the ADM momenta; K. V. Kuchař, J. Math. Phys. 22, 2640 (1981).

25. C. G. Torre, Class. Quantum Grav. 8, 1895 (1991). 